\title{
Current high prevalences of Strongyloides stercoralis and Opisthorchis viverrini infections in rural communities in northeast Thailand and associated risk factors
}

Pokkamol Laoraksawong ${ }^{1}$, Oranuch Sanpool ${ }^{2,5}$, Rutchanee Rodpai ${ }^{2,5}$, Tongjit Thanchomnang ${ }^{3,5}$, Wanida Kanarkard ${ }^{4}$, Wanchai Maleewong ${ }^{2,5}$, Ratthaphol Kraiklang ${ }^{1}$ and Pewpan M. Intapan ${ }^{2,5^{*}}$ (D)

\begin{abstract}
Background: Two important helminths, Strongyloides stercoralis (an intestinal roundworm) and Opisthorchis viverrini (a liver fluke), are endemic in northeast Thailand. There have been national campaigns in place aimed at the control and eradication of soil-transmitted helminthiasis and opisthorchiasis in Thailand for several decades. However, these helminths still exist and raise concerns regarding public health. This study aimed to evaluate the current prevalence of S. stercoralis and O. viverrini infections in rural communities in northeast Thailand. The data from this study will be useful to improve strategies for future helminth prevention and control.
\end{abstract}

Methods: A cross-sectional study was conducted from December 2016 to June 2017 in Mueang Khon Kaen district in Khon Kaen, Thailand. The participants were selected using a simple random sampling method. Demographic data were collected using a questionnaire. Stool samples were collected and processed using agar plate culture to determine the presence of $S$. stercoralis infection and an in-house formalin-ethyl acetate concentration technique to determine the presence of $\mathrm{O}$. viverrini and other intestinal parasite infections (IPIs).

Results: In total, 602 persons were enrolled. However, only 526 were analyzed for S. stercoralis and 387 for 0. viverrini risk factors. The overall prevalence of S. stercoralis infection was $23.0 \%$ (95\% confidence interval [95\%Cl]: 19.4 to 26.6). The prevalence of O. viverrini infection and IPls other than S. stercoralis was 20.4\% (95\%Cl: 16.5 to 24.8$)$. The prevalence of O. viverrini infection was $19.4 \%$ (95\%Cl: 15.6 to 23.7). Male sex was significantly associated with S. stercoralis infection [Adjusted Odds Ratio (aOR) 4.0; 95\%Cl: 2.5 to 6.2; P-value $<0.001$ ]. Males were significantly more likely to be infected with O. viverrini and other IPIs (aOR 4.1; 95\%Cl: 2.3 to $7.2, P$-value $<0.001$ ).

Conclusions: This study demonstrated that the updated prevalence of intestinal parasite infections is still high in rural communities in northeast Thailand, especially that of strongyloidiasis and opisthorchiasis.

Keywords: Prevalence, Intestinal parasites, Strongyloides stercoralis, Opisthorchis viverrini, Thailand

\footnotetext{
* Correspondence: pewpan@kku.ac.th

${ }^{2}$ Department of Parasitology, Faculty of Medicine, Khon Kaen University,

Khon Kaen, Thailand

${ }^{5}$ Research and Diagnostic Center for Emerging Infectious Diseases, Khon

Kaen University, Khon Kaen, Thailand

Full list of author information is available at the end of the article
}

(c) The Author(s). 2018 Open Access This article is distributed under the terms of the Creative Commons Attribution 4.0 International License (http://creativecommons.org/licenses/by/4.0/), which permits unrestricted use, distribution, and reproduction in any medium, provided you give appropriate credit to the original author(s) and the source, provide a link to the Creative Commons license, and indicate if changes were made. The Creative Commons Public Domain Dedication waiver (http://creativecommons.org/publicdomain/zero/1.0/) applies to the data made available in this article, unless otherwise stated. 


\section{Background}

Human strongyloidiasis is a soil-transmitted helminthiasis caused by Strongyloides stercoralis. It is widespread throughout Southeast Asia, including in Thailand [1-5]. It occurs primarily in developing communities and is often found in travelers and former war veterans, immigrants, immunocompromised inhabitants, and people exposed to soil [6]. Some patients presented gastro-intestinal symptoms, dermatological symptoms, hyperinfection, or disseminated strongyloidiasis, which can involve several organs and lead to fatal outcomes [7, 8]. Additionally, chronic strongyloidiasis can lead to malnutrition in children and adolescents, causing growth retardation [7]. The human liver fluke (Opisthorchis viverrini) is a cause of critical public health problems in some areas of Southeast Asia including Lao People's Democratic Republic (Lao PDR), Cambodia, central Vietnam, and Thailand [9]. Opisthorchis viverrini is classified as a Group 1 carcinogen by the World Health Organization (WHO) [10] and is associated with hepatobiliary diseases and the etiological agents of bile duct cancer (cholangiocarcinoma). For several decades there have been intensive national control programs aimed at combating soil-transmitted helminths and opisthorchiasis in rural Thailand $[11,12]$. Moreover, there have been great initiatives put into place to control the $O$. viverrini infection and the resulting cholangiocarcinoma including the EcoHealth/One Health approach (the Lawa Lake control approach) [13] and the Cholangiocarcinoma Screening and Care Program [14]. This study examines the current prevalences of $S$. stercoralis and $O$. viverrini infections in rural communities in the Mueang Khon Kaen district in Khon Kaen, Thailand. Intestinal parasitic infections other than $S$. stercoralis and O. viverrini were also reported. This result is important for monitoring and the implementation of effective control strategies.

\section{Methods}

Study design

This cross-sectional survey was carried out from December 2016 to June 2017 in two sub-districts in Mueang Khon Kaen district in Khon Kaen, Thailand. Mueang Khon Kaen is located at 8.71944 latitude and 99.791667 longitude (Fig. 1). The average temperature was $27.7{ }^{\circ} \mathrm{C}\left(16.0{ }^{\circ} \mathrm{C}-39.9{ }^{\circ} \mathrm{C}\right)$ with an annual rainfall of $1031.4 \mathrm{~mm}$ [15]. Factors associated with the tropical environment in this area (especially education, socio-cultural factors, economic factors, climate, land use, rivers, and rainfall) affect the prevalence of intestinal parasite infection (including that of S. stercoralis and O. viverrini) [16, 17]. The Mueang Khon Kaen District was selected as the study site because it has a higher prevalence of strongyloidiasis than any other district in Khon Kaen [17]. People in this area commonly eat raw, pickled, or undercooked fish and other aquatic animals, which are foods that carry a high risk of O. viverrini infection [18]. We randomly selected villages along the Pong River classified by their varied flooding patterns. Most of the land in two sub-districts is used for farming. Agriculture is the primary economic activity of the population in these areas [15].

\section{Study population, sample size, and sampling technique}

We selected two sub-districts in the Mueang Khon Kaen district to conduct this study: Samran (SR), which had no history of flooding, and Bueng Niam (BN), which experiences at least one flood per year. These areas were selected based on data from the hospital-based study. It was found that the Mueng Khon Kaen district has the highest prevalence of strongyloidiasis than other districts in Khon Kaen [17]. The sample size was determined using the single population proportion formula [19]. It was calculated using a prevalence rate (p) of $23.0 \%$, as detailed in a previous study [1], with a 95\% confidence interval $(\mathrm{z}=1.96)$ and a $5 \%$ margin of error $(\mathrm{d}=0.05)$. The calculated sample size was 273 people per sub-district. We assumed that the final sample size would end up being reduced by around 10\% due to subjects being unable to pass stool on the study date. Thus, we aimed for a sample size of 301 per sub-district. A simple random sampling method was used to select the population from each sub-district. Inclusion criteria were participants who were $\geq 8$ years old and lived in both areas. The individual was randomly selected by the simple random sampling method. We randomly selected 301 people per area and then gave them instructions and distributed plastic containers for stool collection. A total of 589 subjects $(295 / 50.08 \%)$ of the total population of three villages in SR and 49.92\% (294) from that of four villages in BN returned stool specimens (Fig. 2; Fig. 3).

\section{Data collection and laboratory processing}

Field staff visited randomly selected areas of the two sub-districts accompanied by the local public health and primary health care officers center. After receiving informed consent from the participants, authorization from parents or legal guardians of minor participants was obtained. The questionnaire included baseline characteristics, knowledge about S. stercoralis and O. viverrini, and risk behaviors for both parasite infections (contact soil, consume raw fish). A total of 589 participants were interviewed by a self-administered questionnaire, or by parents or legal guardians of minors. Then, stool samples were collected. Before stool-sample collection, a cleaned plastic container labeled with the participant's name and identification was distributed to each participant by the village health volunteers. One day later, they returned the full container to the field staff. The containers were transported immediately to the parasitology laboratory at the Khon Kaen University Faculty of Medicine. Before data 

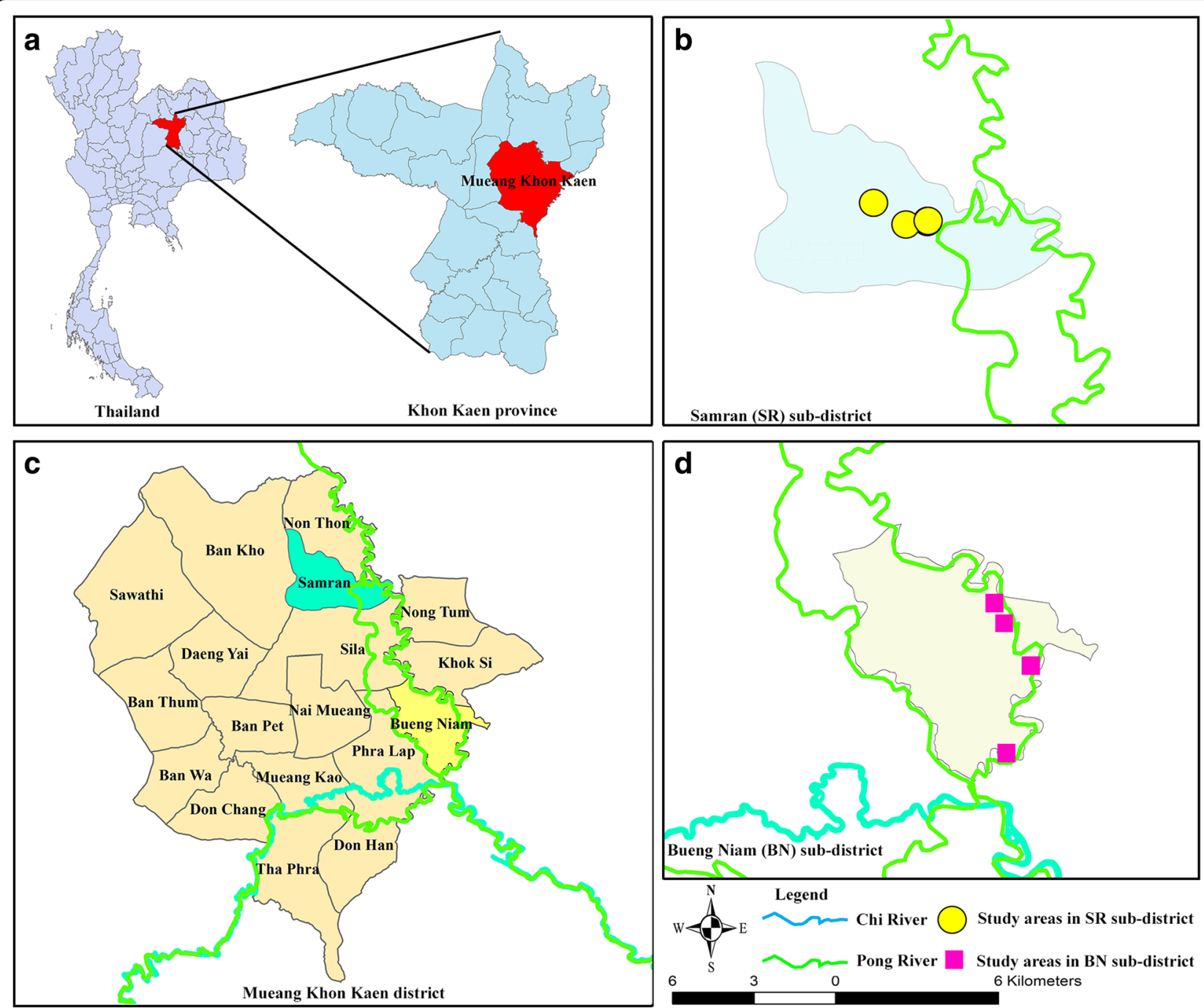

Fig. 1 (a) indicates Khon Kaen province, located in northeast Thailand, and indicates the Mueang Khon Kaen District located in Khon Kaen Province. (b) indicates the Samran (SR) and Bueng Niam (BN) sub-districts, located in Mueang Khon Kaen District. (c and d) indicate areas in the SR and BN sub-districts in which samples were collected, respectively

were analyzed, the data were entered by two researchers for double-checking the quality of the data.

Agar plate culture (APC) [20] was used to detect S. stercoralis in each specimen, and the in-house formalin-ethyl acetate concentration technique (FECT) [21] was used to examine $O$. viverrini and other intestinal parasites. Two to four grams of stool from each sample was placed on $1.5 \%$ nutrient agar plates and incubated at $25-27 \mathrm{C}$ for $3-$ 5 days [20]. The agar plate examination was done under a stereo microscope on the third or fifth day. The formalin-ethyl acetate concentration technique was conducted as follows: two grams of stool from each of the samples was suspended in a container of $10 \mathrm{ml}$ of normal saline solution. The suspension was strained through two layers of gauze into a $15-\mathrm{ml}$ centrifuge tube and centrifuged at $700 \mathrm{x}$ $g$ for five minutes. The supernatant was then decanted. After that, $7 \mathrm{ml}$ of $10 \%$ formalin was added to the sediment, mixed well, and allowed to stand for two minutes. Then, $3 \mathrm{ml}$ of ethyl acetate was added. The tube was closed, vigorously shaken for one minute, and centrifuged at $700 \times g$ for five minutes. The debris plug in the ethyl acetate layer was loosened, and the top three layers were discarded, leaving the sediment. One milliliter of $10 \%$ formalin was then added to re-suspend the sediment, which was examined for parasites under a compound microscope [21].

\section{Data analysis}

Demographic characteristics of the participants are described using the frequency, percentage, mean, and standard deviation (SD). The prevalence of S. stercoralis, O. viverrini, and other intestinal parasitic infections (IPIs) is described as a proportion and 95\%CI. 


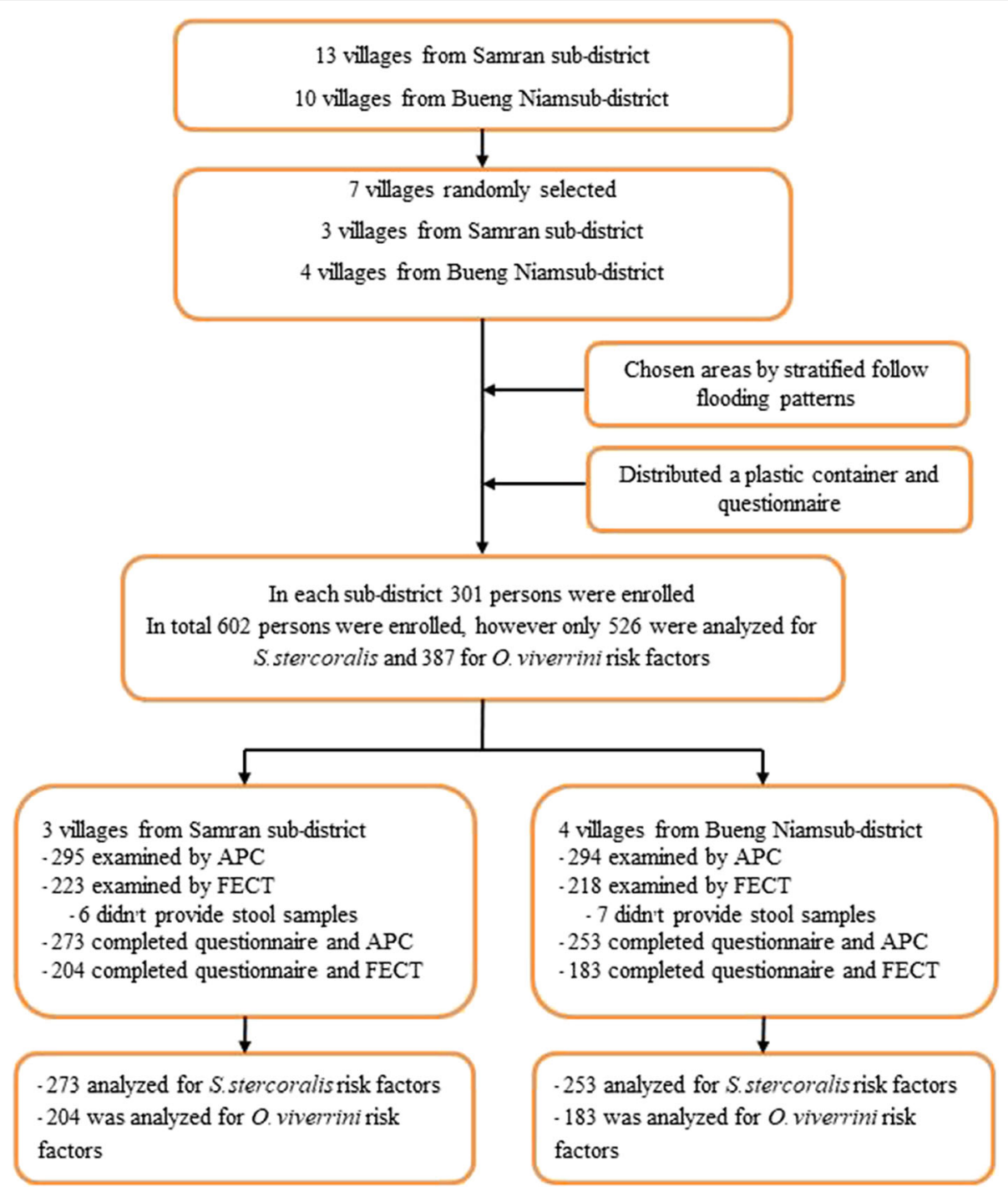

Fig. 2 Flow chart of the study's activities

To investigate factors that affect $S$. stercoralis, $O$. viverrini, and other IPIs, the prevalence rate and their 95\% CIs were estimated using logistic regression and the generalized estimating equation for survey sampling. To adjust for possible confounders, all variables with a $P$-value less than 0.1 in the univariate analysis were selected. A P-value of less than 0.05 was considered statistically significant. The statistical analysis was conducted using the STATA package version 10.1. College Station, Texas: StataCorp LLC.

\section{Results}

\section{Demographic characteristics}

In total, 602 persons were enrolled. However, only 526 were analyzed for S. stercoralis and 387 for O. viverrini risk factors. A total of 526 participants were enrolled in this study (273 [51.9\%] from the SR sub-district, 253 [48.1\%] from the
BN sub-district). Two-hundred fifty-four (48.3\%) of the 526 participants were male, and $272(51.7 \%)$ were female. The mean age $( \pm \mathrm{SD})$ of participants was $55.9( \pm 14.4$; range $=$ $11-91$ years), and most of the participants were $\geq 60$ years old $(246 / 526 ; 46.8 \%)$. The most common occupation of participants was agriculture 368/526 (69.9\%), and the majority had a primary-school education or no formal education $381 / 526(72.4 \%)$. The average income $( \pm$ SD) was $160.4 \pm$ $168.1 \$$ ( 0 to $1500.2 \$$ ), and $397 / 526$ participants $(75.5 \%$ ) had an income lower than $\$ 250$ per month (according to the USD-THB exchange rate as of 1 November 2017), which is the poverty line in Thailand [22] (Table 1).

Prevalence of S. stercoralis infection as detected using the APC technique

The overall prevalence of $S$. stercoralis infection as detected using APC was 121/526 participants (23.0\%; 


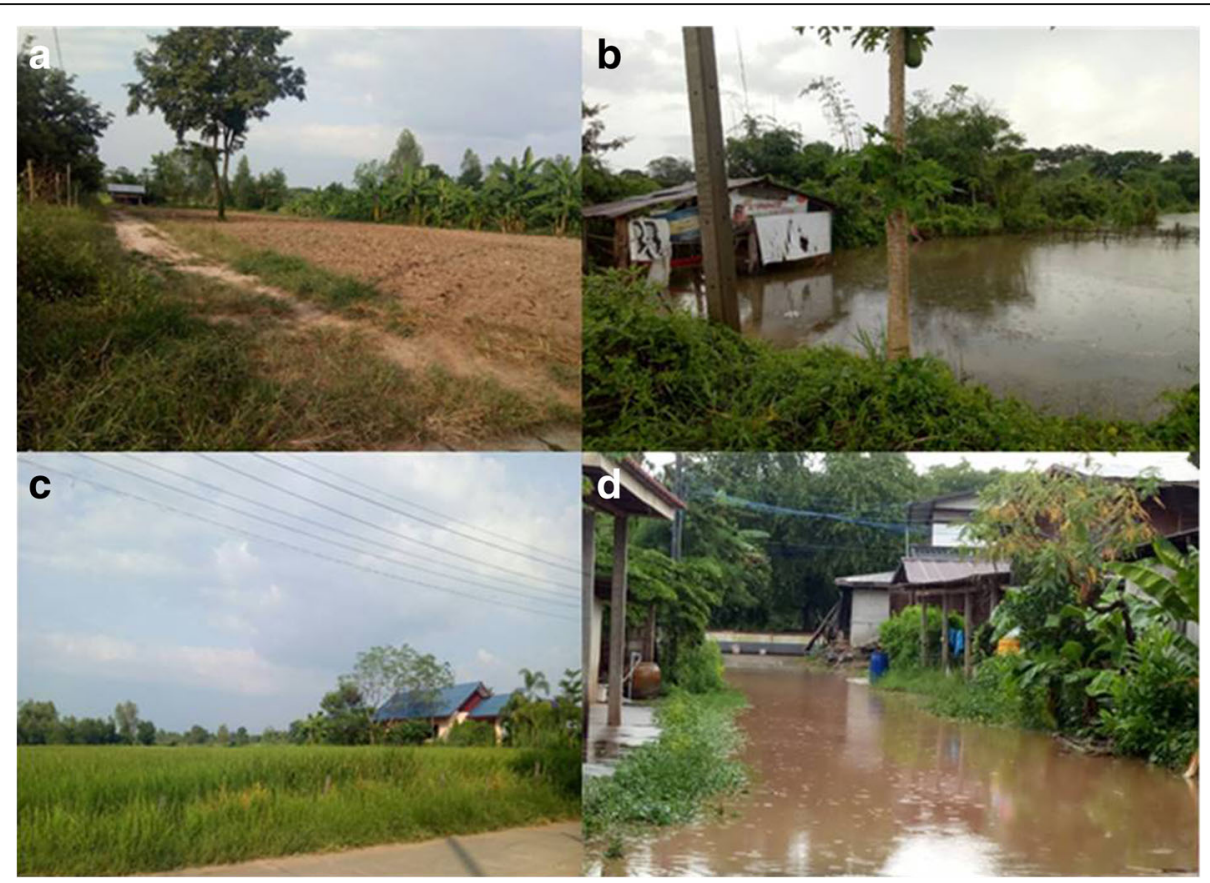

Fig. 3 Geographic characteristics of Samran (SR) and Bueng Niam (BN) sub-districts; a and $\mathbf{c}$ show the geographic characteristics of the SR sub-district during the rainy season, $\mathbf{b}$ and $\mathbf{d}$ show the geographic characteristics of the BN sub-district during the rainy season

95\%CI: 19.4 to 26.6$), 88$ (16.7\%) of whom were male and $33(6.3 \%)$ of whom were female. Most of the participants infected with S. stercoralis $(95 / 526 ; 18.0 \%)$ had a primary-school level or no formal education. Moreover, those working in agriculture had a higher prevalence of $S$. stercoralis infection than those in other occupations (95/526; 18.0\%). Most participants infected with S. stercoralis had an income of less than $\$ 250.00$ per month (97/526; 18.4\%) (Table 2). In the SR sub-district, the prevalence of S. stercoralis infection was 61/273 (22.3\%; 95\% CI: 17.5 to 27.8$)$. Forty-one (15.0\%) of the people infected in this sub-district were male, and 20 (7.3\%) were female. In the BN sub-district, the prevalence of $S$. stercoralis infection was 60/253 (23.7\%; 95\%CI: 18.6 to 29.4) participants, $47(18.6 \%)$ of whom were male and $13(5.1 \%)$ of whom were female. The prevalence of $S$. stercoralis infection increased with age in the overall and both sub-district samples (Table 2). The difference in prevalence of $S$. stercoralis infection between the two sub-districts was not statistically significant.

\section{Risk factors associated with S. stercoralis infection}

According to univariate analysis, demographic characteristics associated with $S$. stercoralis infection were sex, age, education level, and occupation. Males were 3.8 times more likely to be infected with $S$. stercoralis than females (crude Odds Ratio [cOR] 3.8; 95\%CI: 2.45 to $5.99, P$-value $<0.001)$. Patients 50-59 years of age and $\geq$ 60 years were 9.9 and 9.5 times more likely to be infected with S. stercoralis than those of other age groups, respectively (cOR 9.9; 95\%CI: 1.29 to 75.47 , cOR 9.5; 95\%CI: 1.26 to $71.29, P$-value $<0.003$, respectively). Participants with a primary school level or no formal education were 4.5 times more likely than those with a diploma, bachelor's degree, or higher to be infected with S. stercoralis (cOR 4.5; 95\%CI: 1.04 to $19.21, P$-value $=$ 0.037). Additionally, agriculturists were 5.2 time more likely to be infected with $S$. stercoralis than participants in other occupations (cOR 5.2; 95\%CI: 1.22 to 22.25, $P$-value $=0.009)$. Body mass index and income were not significantly associated with $S$. stercoralis infection $(P$-value $>0.05)$. After multivariable analysis to adjust for possible confounders, gender and age were found to be risk factors associated with $S$. stercoralis infection. Males were 4.0 times more likely to be infected with S. stercoralis than females (aOR 4.0; 95\%CI: 2.52 to 6.24, P-value $<0.001$; Table 2).

\section{Prevalence of $O$. viverrini and IPIs other than S. stercoralis detected using FECT}

The 387 stool samples that remained after APC examination were subjected to FECT. The overall prevalence of O. viverrini and IPIs other than S. stercoralis was 79/387 (20.4\%; 95\%CI: 16.5 to 24.8 ). The highest prevalence of infection was of $O$. viverrini infection, which was $75 / 387$ (19.4\%; 95\%CI: 15.6 to 23.7), followed by Taenia spp., which was 5/387 (1.3\%; 95\%CI: 0.4 to 3.0 ; Fig. 4$)$. In the SR sub-district, the prevalence of $O$. viverrini and other 
Table 1 Demographic characteristics of study participants in Mueang Khon Kaen district, Khon Kaen province, Thailand

\begin{tabular}{|c|c|c|c|c|c|c|}
\hline \multirow[t]{2}{*}{ Demographic data } & \multicolumn{2}{|l|}{$\underline{S R}$} & \multicolumn{2}{|l|}{$\mathrm{BN}$} & \multicolumn{2}{|l|}{ Total } \\
\hline & Number & Percentage & Number & Percentage & Number & Percentage \\
\hline Gender (N) & 273 & & 253 & & 526 & \\
\hline Female & 144 & 52.8 & 128 & 50.6 & 272 & 51.7 \\
\hline Male & 129 & 47.2 & 125 & 49.4 & 254 & 48.3 \\
\hline Age $(\mathrm{N})$ & 273 & & 253 & & 526 & \\
\hline$<30$ & 18 & 6.6 & 10 & 4.0 & 28 & 5.3 \\
\hline $30-39$ & 17 & 6.2 & 12 & 4.7 & 29 & 5.5 \\
\hline $40-49$ & 45 & 16.5 & 51 & 20.2 & 96 & 18.3 \\
\hline $50-59$ & 68 & 24.9 & 59 & 23.3 & 127 & 24.1 \\
\hline$\geq 60$ & 125 & 45.8 & 121 & 47.8 & 246 & 46.8 \\
\hline Mean \pm SD (range) & \multicolumn{2}{|c|}{$55.6 \pm 14.8(11$ to 91$)$} & \multicolumn{2}{|c|}{$56.2 \pm 14.0(12$ to 80$)$} & \multicolumn{2}{|c|}{$55.9 \pm 14.4(11$ to 91$)$} \\
\hline Education level (N) & \multicolumn{2}{|l|}{273} & \multicolumn{2}{|l|}{253} & \multicolumn{2}{|l|}{526} \\
\hline Diploma, bachelor degree or higher & 17 & 6.2 & 12 & 4.7 & 29 & 5.5 \\
\hline Grade $7-12$ & 57 & 20.9 & 59 & 23.3 & 116 & 22.1 \\
\hline Primary school or no formal education & 199 & 72.9 & 182 & 72.0 & 381 & 72.4 \\
\hline Occupation (N) & \multicolumn{2}{|l|}{273} & \multicolumn{2}{|l|}{253} & \multicolumn{2}{|l|}{526} \\
\hline Student, government/private office & 24 & 8.8 & 8 & 3.2 & 32 & 6.1 \\
\hline Employee/Merchant/older & 93 & 34.1 & 33 & 13.0 & 126 & 24.0 \\
\hline Agriculturist & 156 & 57.1 & 212 & 83.8 & 368 & 69.9 \\
\hline BMI (N) & \multicolumn{2}{|l|}{259} & \multicolumn{2}{|l|}{245} & \multicolumn{2}{|l|}{504} \\
\hline$<18.5$ & 22 & 8.5 & 9 & 3.7 & 31 & 6.2 \\
\hline 18.5 to 25.9 & 140 & 54.1 & 136 & 55.5 & 276 & 54.7 \\
\hline 26 to 29.9 & 78 & 30.1 & 79 & 32.2 & 157 & 31.2 \\
\hline$\geq 30$ & 19 & 7.3 & 21 & 8.6 & 40 & 7.9 \\
\hline Mean \pm SD (range) & \multicolumn{2}{|c|}{$24.0 \pm 4.2(12.9$ to 38.1$)$} & \multicolumn{2}{|c|}{$24.5 \pm 4.1$ (16.8 to 50.2$)$} & \multicolumn{2}{|c|}{$24.2 \pm 4.2(12.9$ to 50.2$)$} \\
\hline Income per month $(\mathrm{N})$ \$ & \multicolumn{2}{|l|}{273} & \multicolumn{2}{|l|}{253} & \multicolumn{2}{|l|}{526} \\
\hline$<250 \$$ & 192 & 70.3 & 205 & 81.0 & 397 & 75.5 \\
\hline$\geq 250 \$$ & 81 & 29.7 & 48 & 19.0 & 129 & 24.5 \\
\hline Mean \pm SD (range) & \multicolumn{2}{|c|}{$165.1 \pm 179.8(0$ to 1200.1$)$} & \multicolumn{2}{|c|}{$155.2 \pm 154.6(0$ to 1515.2$)$} & \multicolumn{2}{|c|}{$160.4 \pm 168.1$ (0 to 1500.2 ) } \\
\hline
\end{tabular}

SR Samran sub-district, $B N$ Bueng Niam sub-district

IPIs was $45 / 204(22.1 \%)$. In the BN sub-district, the prevalence of $O$. viverrini and other IPIs was $34 / 183$ (18.6\%). The prevalence of O. viverrini and other IPIs did not differ significantly between the two sub-districts (Table 3).

\section{Risk factors associated with $O$. viverrini and IPIs other than S. stercoralis}

Males were more likely to be infected with $O$. viverrini than females $(49 / 387 ; 12.7 \%$ vs. $30 / 387 ; 7.7 \%)$. There was a higher prevalence of infection in participants $\geq 60$ years than in other age groups $(45 / 387 ; 11.6 \%)$. Prevalence was highest among participants with primary level or no formal education $(68 / 387 ; 17.6 \%)$. Among the various occupations, participants who worked in agriculture were most likely to be infected (57/387; $14.7 \%$; Table 3 ).
According to the univariate analysis, gender and education level were significantly associated with $O$. viverrini and IPIs other than S. stercoralis. Males were 2.4 times more likely to be infected with $O$. viverrini and IPIs than females (cOR 2.4; 95\%CI: 1.4 to 3.9, $P$-value $<0.001$ ). Participants with primary level or no formal education were 2.3 times more likely to be infected with $O$. viverrini or IPIs other than $S$. stercoralis than those who had completed grades $7-12$ or had a diploma, bachelor's degree, or higher (cOR 2.3; 95\%CI: 0.5 to $10.5, P$-value $=$ 0.005). Age, occupation, BMI, and income were not significantly associated with $O$. viverrini infection or IPIs other than $S$. stercoralis ( $P$-value $>0.05$; Table 3 ). After multivariable analysis to adjust for possible confounders, only gender was a risk factor that was associated with $O$. viverrini infection or IPIs other than S. stercoralis. Males 


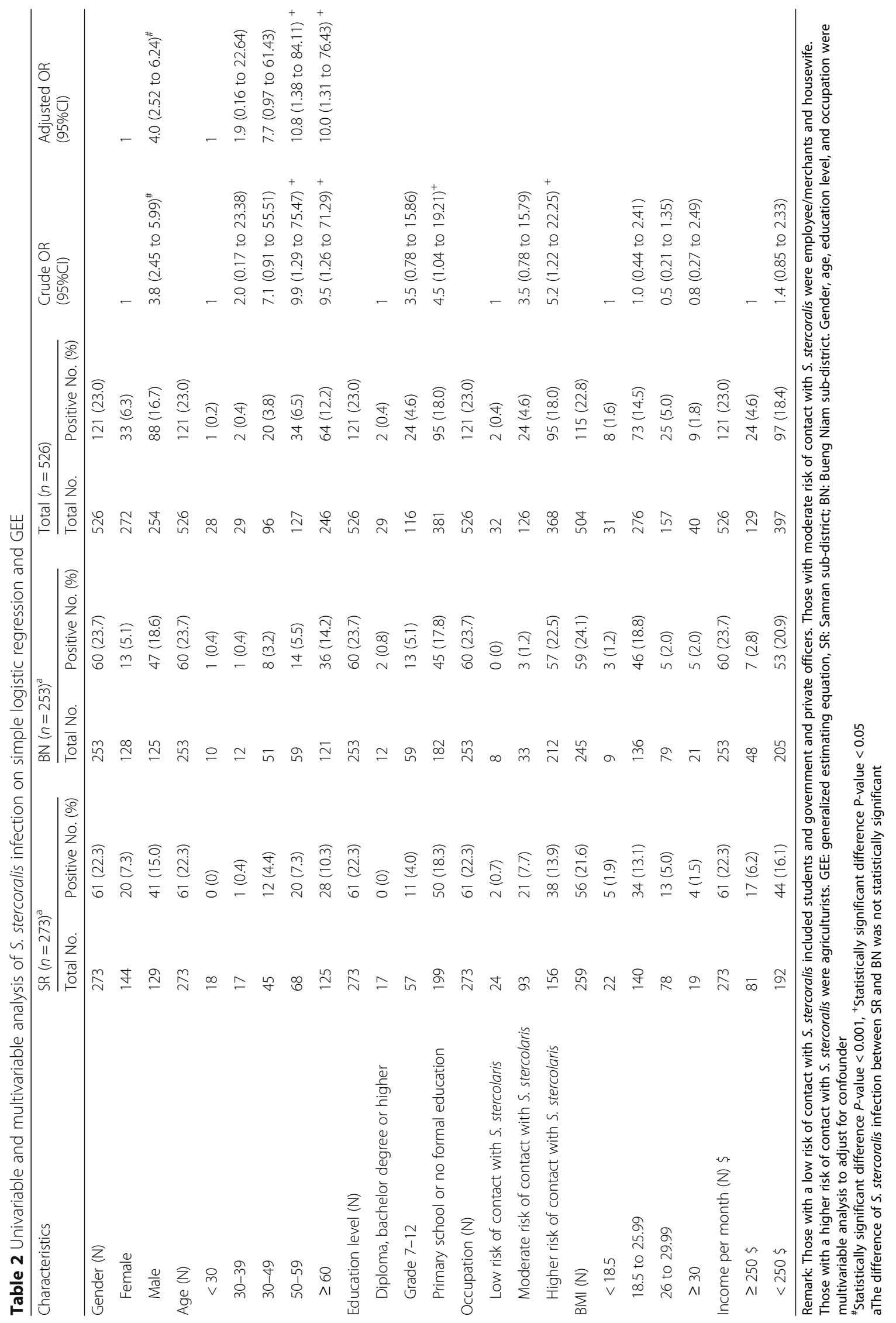




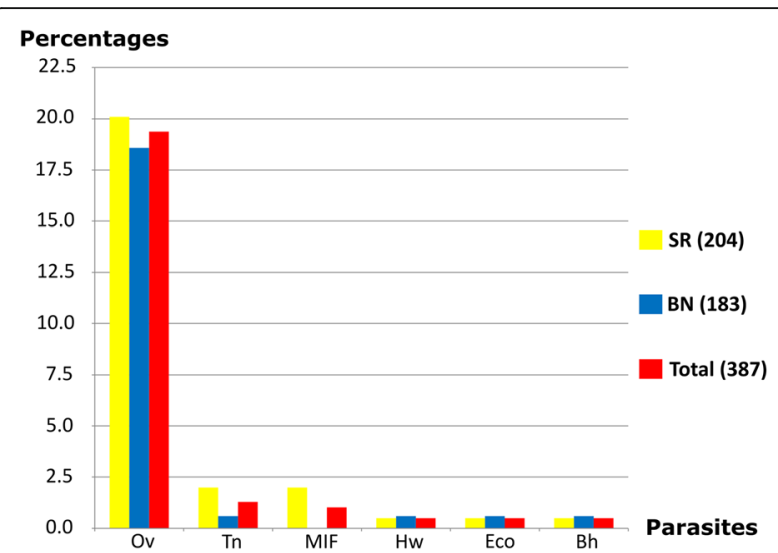

Fig. 4 Prevalence of intestinal parasitic infections except Strongyloides stercoralis examined using FECT in Mueang Khon Kaen district, Khon Kaen province, Thailand, classified by parasite. Remark: Bh; Blastocystis hominis, Eco; Entamoeba coli, Hw; Hookworms, MIF; Minute Intestinal Flukes, Ov; Opisthorchis viverrini, Tn; Taenia spp. FECT: formalin ethyl acetate concentration technique

were 4.1 times more likely than females to be infected with $O$. viverrini and IPIs other than S. stercoralis (aOR 4.1; 95\%CI: 2.3 to $7.2, P$-value $<0.001$; Table 3 ).

\section{Discussion}

This study demonstrated a high prevalence of S. stercoralis and $O$. viverrini infections in rural communities in northeast Thailand's Mueang Khon Kaen district. The overall prevalence of $S$. stercoralis infection as detected by APC of $23.0 \%$ was similar to those found in past studies of the same area, reflecting no decline. For instance, a previous survey in northeast Thailand found rates of infection to be 22.7, 23.52, and 28.9\% [23-25]. Moreover, the prevalence of $S$. stercoralis infection in this study was higher than in some previous studies. Surveys using the same APC method in northern and southern Thailand found the prevalence to be $15.9 \%$ [26] and 20.6\% [27], respectively. Other surveys of $S$. stercoralis human infection using FECT, the Kato-Katz technique, and the Harada-Mori technique in northeast Thailand found the prevalence to be from 2.8 to $9.5 \%$ [28-30]. The difference in prevalence of S. stercoralis among various communities may be due to variations in age, the season of study, the parasitological technique used, personal hygiene practices, and socioeconomic and environmental factors [31-33].

The present study showed various factors (including gender, age, education levels, and occupation) to be significantly associated with $S$. stercoralis infection. We found that males were more likely to be infected with $S$. stercoralis than females $(16.7 \%$ vs. $6.3 \%)$, which was similar to the results found in previous reports [34-39]. This study found that older participants ( $\geq 60$ years) had the highest prevalence of $S$. stercoralis infection (12.2\%), which was also similar to the results of previous studies [40]. This is possibly due to older participants having a more prolonged exposure to sources of S. stercoralis infection [17, 27]. Most people who were infected with $S$. stercoralis had primary school or no formal education (18.0\%). This is consistent with the findings of a previous report [33]. The highest prevalence of S. stercoralis infection was in agriculturists $(18.0 \%)$, which indicates that this group is at high risk of coming in contact with $S$. stercoralis in its infective stage $[35,38]$. The reason may be simply because they have greater contact with soil than those in other occupations.

In this study, the prevalence of O. viverrini and IPIs other than S. stercoralis detected by FECT was 20.4\%, which was lower than in a previous study in which it was $37.2 \%$ [28] and a national survey in the northeast of Thailand that found $26.0 \%$ [30]. However, the prevalence of $O$. viverrini and IPIs other than S. stercoralis in this study was higher than in other studies. For instance, a survey using the Kato-Katz technique in northeast Thailand found a prevalence of 7.0\% [29], and one in northern Thailand found a prevalence of 5.1\% [26]. Moreover, a survey using FECT in northeast Thailand found a prevalence of 5.4\% [25]. The differences in prevalences may be due to variations in examination technique, environmental sanitation, socioeconomic factors, and the education level of participants.

The prevalence of $O$. viverrini infection was $19.4 \%$, which was higher than that of other helminths. This finding was similar to those in previous studies. For example, a national survey revealed an O. viverrini infection in the northeast in 2001 and 2009 with a prevalence of 15.7 and $16.6 \%$, respectively, which is higher than in other regions [12, 27, 41]. Additionally, a study in rural communities in northeast Thailand showed that $O$. viverrini $(26.9 \%)$ was a prominent intestinal parasite in this region [28]. Raw fish consumption is common in northeast Thailand and may be related to poverty [27, 28]. A previous report found Clonorchis sinensis in central Thailand using a molecular method [42]. Opisthorchis viverrini and C. sinensis are virtually indistinguishable by egg morphology. This means that microscopic examination may result in an $O$. viverrini infection being misinterpreted for a $C$. sinensis infection. Thus, it is possible that perhaps clonorchiasis and opisthorchiasis may be present in this area. This possibility needs to be tested empirically in the future.

This study found that gender and education levels were significantly associated with $O$. viverrini and IPIs other than S. stercoralis. Males had a greater chance of being infected with $O$. viverrini and IPIs other than $S$. stercoralis than females ( $12.7 \%$ vs. $7.7 \%)$, which is similar to the findings of previous reports [28]. Most people infected with O. viverrini and IPIs other than S. stercoralis 


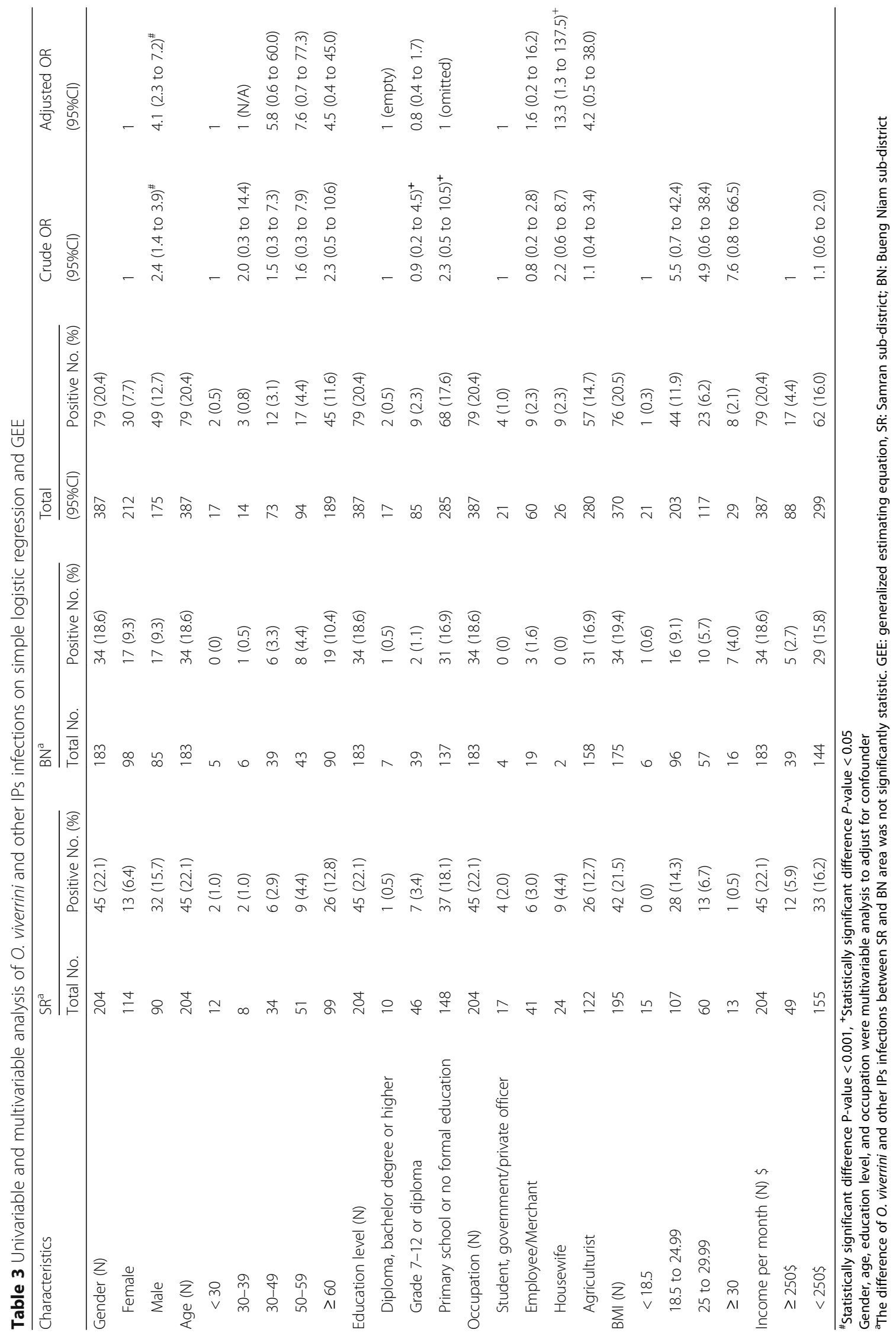


were primary school graduates or had no formal education (17.6\%). These findings were similar to previous reports [28]. Participants $\geq 60$ years old had the highest prevalence of $O$. viverrini and IPI infections other than S. stercoralis $(11.6 \%)$, similar to the results found in previous studies $[28,34]$. However, this differs from the results of a national survey, which found the highest infection rates among 40-49-year-olds [27]. The fact that older participants had a high prevalence of $O$. viverrini and S. stercoralis may be due to them having more time to be exposed to sources of $S$. stercoralis infection and having consumed a greater amount of raw fish (a source of $O$. viverrini infection).

When considering the prevalence of $S$. stercoralis infection $(22.3 \%$ ) in the SR sub-district (an area in which there was no history of flooding) and the $\mathrm{BN}$ sub-district (23.7\%; at least one flood per year), the prevalence in the two areas did not differ significantly. This result differs from those of a previous laboratory experiment [43]. Ananmnart et al. [43] demonstrated that prolonged submersion of stool in water was detrimental to the growth and development of S. stercoralis rhabditiform larva and suggested that atmospheric conditions and rainfall could possibly affect the growth and development of $S$. stercoralis. This effect is absent in the present study, possibly due to other factors (i.e., poverty, climatic conditions, poor personal hygiene, poor sanitation, population migration, and consumption of raw or semi-raw meat).

\section{Conclusions}

This study demonstrated that, despite the implementation of an intensive national parasite control program $[11,12]$, a sustainable integrated opisthorchiasis control program [13], as well as a cholangiocarcinoma screening and care program [14] in rural areas of northeast Thailand, the prevalences of S. stercoralis and O. viverrini have not declined. Another nationwide assessment of intestinal parasitic examination including risk factors, treatment, and prevention should be conducted. Moreover, awareness campaigns and appropriate control programs should be developed to reduce intestinal parasitic infection, especially in agriculturists in rural communities. Additionally, these results should encourage policymakers and public health personnel to improve programs for parasitic control and health promotion.

\footnotetext{
Abbreviations

\$: US Dollar; 95\%Cl: 95\% confidence interval; aOR: adjusted odds ratio; APC: Agar plate culture; BMl: Body mass index; BN: Bueng Niam sub-district; COR: Crude odds ratio; FECT: Formalin-ethyl acetate concentration technique; GEE: Generalized estimating equation; IPIs: Intestinal parasitic infections; Lao PDR: Lao People's Democratic Republic; ${ }^{\circ} \mathrm{C}$ : Degrees Celsius; SD: Standard deviation; SR: Samran sub-district; WHO: World Health Organization
}

\section{Acknowledgements}

We wish to acknowledge the support of the English Consultation Clinic at the Khon Kaen University Faculty of Medicine Research Affairs Division and the Khon Kaen University Publication Clinic at the Research and Technology Transfer Affairs Division for their assistance. The authors would like to acknowledge the Directors of Mueang district Public Health Office and the Directors of each sub-district health promoting hospital for their support throughout the course of this study.

\section{Funding}

This study was supported by a TRF Senior Research Scholar Grant, Thailand Research Fund grant number RTA5880001; OS was supported by the Scholarship under the Post-Doctoral Training Program from Research Affairs and Graduate School, Khon Kaen University (58101). The findings and conclusions in this report are those of the authors and do not necessarily represent the official position of the funding agencies.

\section{Availability of data and materials}

The datasets used and/or analyzed during the current study are available from the corresponding author on reasonable request.

\section{Authors' contributions}

All authors conceived of the idea and participated in the design of this study. PL, RR, OS and TT conducted the study. PL, WM, WK, PM, and RK were responsible for interpretation of the results and drafting of the manuscript and reading for intellectual content. All authors read and approved the final manuscript.

\section{Ethics approval and consent to participate}

Ethical approval was obtained from the Khon Kaen University Ethics Committee for Human Research (HE 591213). All research was performed in accordance with the Declaration of Helsinki. The aim of the study was fully explained to the participants, and participation was voluntary. Written informed consent was obtained from all participants before data and sample collection.

\section{Consent for publication}

Not applicable.

\section{Competing interests}

The authors declare that they have no competing interests.

\section{Publisher's Note}

Springer Nature remains neutral with regard to jurisdictional claims in published maps and institutional affiliations.

\section{Author details}

'Department of Public Health Administration, Health Promotion, Nutrition, Faculty of Public Health, Khon Kaen University, Khon Kaen, Thailand. 2Department of Parasitology, Faculty of Medicine, Khon Kaen University, Khon Kaen, Thailand. ${ }^{3}$ Faculty of Medicine, Mahasarakham University, Mahasarakham, Thailand. ${ }^{4}$ Department of Computer Engineering, Faculty of Engineering, Khon Kaen University, Khon Kaen, Thailand. ${ }^{5}$ Research and Diagnostic Center for Emerging Infectious Diseases, Khon Kaen University, Khon Kaen, Thailand.

Received: 2 February 2018 Accepted: 20 July 2018

Published online: 31 July 2018

\section{References}

1. Jongsuksuntigul P, Intapan PM, Wongsaroj T, Nilpan S, Singthong $S$, Veerakul S, et al. Prevalence of Strongyloides stercoralis infection in northeastern Thailand (agar plate culture detection). J Med Assoc Thail. 2003;86:737-41.

2. Jongwutiwes $U$, Waywa $D$, Silpasakorn $S$, Wanachiwanawin $D$, Suputtamongkol Y. Prevalence and risk factors of acquiring Strongyloides stercoralis infection among patients attending a tertiary hospital in Thailand. Pathog Glob Health. 2014;108:137-40.

3. Nutman TB. Human infection with Strongyloides stercoralis and other related Strongyloides species. Parasitology. 2016:1-11.

4. Olsen A, van Lieshout L, Marti H, Polderman T, Polman K, Steinmann P, et al. Strongyloidiasis--the most neglected of the neglected tropical diseases? Trans R Soc Trop Med Hyg. 2009;103:967-72. 
5. Schär F, Trostdorf U, Giardina F, Khieu V, Muth S, Marti H, et al. Strongyloides stercoralis: global distribution and risk factors. PLoS Negl Trop Dis. 2013;7:e2288.

6. Beknazarova M, Whiley $H$, Ross K. Strongyloidiasis: A Disease of Socioeconomic Disadvantage. Int J Environ Res Public Health. 2016;13: pii: E517.https://doi.org/10.3390/ijerph13050517

7. Forrer A, Khieu V, Schar F, Hattendorf J, Marti H, Neumayr A, et al. Strongyloides stercoralis is associated with significant morbidity in rural Cambodia, including stunting in children. PLoS Negl Trop Dis. 2017;11(10): e0005685.

8. Grove DI. Human strongyloidiasis. Adv Parasitol. 1996;38:251-309.

9. Sripa B, Kaewkes S, Intapan PM, Maleewong W, Brindley PJ. Food-borne trematodiases in Southeast Asia epidemiology, pathology, clinical manifestation and control. Adv Parasitol. 2010;72:305-50.

10. Bouvard V, Baan R, Straif K, Grosse Y, Secretan B, Ghissassi F, et al. WHO International Agency for Research on Cancer monograph working group. A review of human carcinogens-part B: biological agents Lancet Oncol. 2009; 10:321-2.

11. Chongsuvivatwong V, Pas-Ong S, Ngoathammatasna W, McNeil D, Vithsupakorn K, Bridhikitti V, et al. Evaluation of hookworm control program in southern Thailand. Southeast Asian J Trop Med Public Health. 1994;25 745-51.

12. Jongsuksuntigul P, Imsomboon T. Opisthorchiasis control in Thailand. Acta Trop. 2003:88:229-32.

13. Sripa B, Tangkawattana S, Laha T, Kaewkes S, Mallory FF, Smith JF, Wilcox BA. Toward integrated opisthorchiasis control in northeast Thailand: the Lawa project. Acta Trop. 2015;141(Pt B):361-7.

14. Khuntikeo N, Chamadol N, Yongvanit P, Loilome W, Namwat N, Sithithaworn P, Andrews RH, Petney TN, Promthet S, Thinkhamrop K, Tawarungruang C, Thinkhamrop B. CASCAP investigators. Cohort profile: cholangiocarcinoma screening and care program (CASCAP). BMC Cancer. 2015;15:459

15. Office of Agriculture Economics, Ministry of Agriculture and Cooperatives. 2016. http://www.oae.go.th/assets/portals/1/files/ebook/yearbook59.pdf. Accessed 6 Sep 2017.[In Thai].

16. Khuntikeo N, Sithithaworn P, Loilom W, Namwat N, Yongvanit P, Thinkhamrop B, et al. Changing patterns of prevalence in Opisthorchis viverrini sensu lato infection in children and adolescents in Northeast Thailand. Acta Trop. 2016:164:469-72.

17. Prasongdee TK, Laoraksawong P, Kanarkard W, Kraiklang R, Sathapornworachai K, Naonongwai $\mathrm{S}$, et al. An eleven-year retrospective hospital-based study of epidemiological data regarding human strongyloidiasis in Northeast Thailand. BMC Infect Dis. 2017;17:627.

18. Saenna P, Hurst C, Echaubard P, Wilcox BA, Sripa B. Fish sharing as a risk factor for Opisthorchis viverrini infection: evidence from two villages in North-Eastern Thailand. Infect Dis Poverty. 2017;6:66.

19. Pennsylvania State University, Sample Size Computation for Population Proportion Confidence Interval. 2016. https://onlinecourses.science.psu.edu/ stat500/node/31. Accessed 6 Sep 2016.

20. Koga K, Kasuya S, Khamboonruang C, Sukhavat K, leda M, Takatsuka N, et al. A modified agar plate method for detection of Strongyloides stercoralis. Am J Trop Med Hyg. 1991;45:518-21.

21. Elkins DB, Haswell-Elkins $M$, Anderson RM. The epidemiology and control of intestinal helminths in the Pulicat Lake region of southern India. I. Study design and pre- and post-treatment observations on Ascaris lumbricoides infection. Trans R Soc Trop Med Hyg. 1986:80:774-92.

22. The Secretariat of the Cabinet. Measures to increase income for low income people. 2016. https://www.mof.go.th/home/Press_release/News2016/154. pdf. Accessed 16 Apr 2018. [In Thai].

23. Intapan PM, Maleewong W, Wongsaroj T, Singthong S, Morakote N. Comparison of the quantitative formalin ethyl acetate concentration technique and agar plate culture for diagnosis of human strongyloidiasis. $J$ Clin Microbiol. 2005:43:1932-3.

24. Sithithaworn J, Sithithaworn P, Janrungsopa T, Suvatanadecha K, Ando K, Haswell-Elkins MR. Comparative assessment of the gelatin particle agglutination test and an enzyme-linked immunosorbent assay for diagnosis of strongyloidiasis. J Clin Microbiol. 2005;43:3278-82.

25. Sithithaworn P, Srisawangwong T, Tesana S, Daenseekaew W, Sithithaworn J, Fujimaki Y, et al. Epidemiology of Strongyloides stercoralis in north-East Thailand: application of the agar plate culture technique compared with the enzyme-linked immunosorbent assay. Trans Roy Soc Trop Med Hyg. 2003; 97:398-402.
26. Nontasut P, Muennoo C, Sa-nguankiat S, Fongsri S, Vichit A. Prevalence of strongyloides in northern Thailand and treatment with ivermectin vs albendazole. Southeast Asian J Trop Med Public Health. 2005;36:442-4.

27. Wongsaroj T, Phatihatakorn W, Ramasoota P, Anamnart W, Kaewpoonsri N, Chiewchanyon B. Epidemiological study of strongyloidiasis in southern Thailand, 2007. J Trop Med Parasitol. 2008;31:6-13.

28. Boonjaraspinyo S, Boonmars T, Kaewsamut B, Ekobol N, Laummaunwai P, Aukkanimart $R$, et al. A cross-sectional study on intestinal parasitic infections in rural communities, Northeast Thailand. Korean J Parasitol. 2013:51:727-34.

29. Kitvatanachai S, Boonslip S, Watanasatitarpa S. Intestinal parasitic infections in Srimum suburban area of Nakhon Ratchasima Province. Thailand Trop Biomed. 2008;25:237-42.

30. Wongsaroj T, Nithikathkul C, Rojkitikul W, Nakai W, Royal L, Rammasut P. Brief communication (Original). National survey of helminthiasis in Thailand. Asian Biomedicine. 2014:779.

31. Hotez PJ, Brindley PJ, Bethony JM, King CH, Pearce EJ, Jacobson J. Helminth infections: the great neglected tropical diseases. J Clin Invest. 2008;118: 1311-21.

32. Hotez PJ, Bundy DAP, Beegle K, Brooker S, Drake L, de Silva N, et al. Helminth infections: soil-transmitted helminth infections and schistosomiasis. In: Jamison DT, Breman JG, Measham AR, Alleyne G, Claeson M, Evans DB, et al., editors. Disease control priorities in developing countries. Washington (DC): World Bank; 2006

33. Punsawad C, Phasuk N, Bunratsami S, Thongtup K, Siripakonuaong N, Nongnaul S. Prevalence of intestinal parasitic infection and associated risk factors among village health volunteers in rural communities of southern Thailand. BMC Public Health. 2017;17:564.

34. Gonçalves $A Q$, Junqueira ACV, Abellana R, PCd B, WCM T, Sodré FC, et al. Prevalence of intestinal parasites and risk factors for specific and multiple helminth infections in a remote city of the Brazilian Amazon Rev Soc Bras Med Trop. 2016:49:119-24.

35. Khieu V, Schär F, Forrer A, Hattendorf J, Marti H, Duong S, et al. High prevalence and spatial distribution of Strongyloides stercoralis in rural Cambodia. PLoS Negl Trop Dis. 2014;8:e2854.

36. Khieu V, Schär F, Marti H, Bless PJ, Char MC, Muth S, et al. Prevalence and risk factors of Strongyloides stercoralis in Takeo Province. Cambodia Parasit Vectors. 2014;7:221.

37. Laymanivong $S$, Hangvanthong B, Insisiengmay B, Vanisaveth $V$, Laxachack $P$, Jongthawin J, et al. First molecular identification and report of genetic diversity of Strongyloides stercoralis, a current major soil-transmitted helminth in humans from Lao People's Democratic Republic. Parasitol Res. 2016;115:2973-80.

38. Steinmann $P$, Zhou XN, Du ZW, Jiang JY, Wang LB, Wang XZ, et al. Occurrence of Strongyloides stercoralis in Yunnan Province, China, and comparison of diagnostic methods. PLoS Negl Trop Dis. 2007;1:e75.

39. Vonghachack Y, Sayasone S, Bouakhasith D, Taisayavong K, Akkavong K, Odermatt P. Epidemiology of Strongyloides stercoralis on Mekong islands in southern Laos. Acta Trop. 2015;141:289-94.

40. Paula F, Costa-Cruz J. Epidemiological aspects of strongyloidiasis in Brazil. Parasitology. 2011:138:1331-40.

41. Sithithaworn $P$, Andrews RH, Nguyen VD, Wongsaroj T, Sinuon M, Odermatt $P$, et al. The current status of opisthorchiasis and clonorchiasis in the Mekong Basin. Parasitol Int. 2012;61:10-6.

42. Traub RJ, Macaranas J, Mungthin M, Leelayoova S, Cribb T, Murrell KD, Thompson RC. A new PCR-based approach indicates the range of Clonorchis sinensis now extends to Central Thailand. PLoS Negl Trop Dis 2009;3(1):e367.

43. Anamnart W, Intapan PM, Pattanawongsa A, Chamavit P, Kaewsawat S, Maleewong W. Effect of dilution of stool soluble component on growth and development of Strongyloides stercoralis. Sci Rep. 2015;5:10749. 